\title{
Popular Integration Grids Can Result in Large Errors in DFT-Computed Free Energies
}

\author{
Andrea N. Bootsma and Steven E. Wheeler* \\ Center for Computational Quantum Chemistry, Department of Chemistry, \\ University of Georgia, Athens, GA 30602 \\ E-mail: swheele2@uga.edu
}

\begin{abstract}
Density functional theory (DFT) has emerged as a powerful tool for analyzing organic and organometallic systems and proved remarkably accurate in computing the small free energy differences that underpin many chemical phenomena (e.g. regio- and stereoselective reactions). We show that the lack of rotational invariance of popular DFT integration grids reveals large uncertainties in computed free energies for isomerizations, torsional barriers, and regio- and stereoselective reactions. The result is that predictions based on DFT-computed free energies for many systems can change qualitatively depending on molecular orientation. For example, for a metal-free propargylation of benzaldehyde, predicted enantioselectivities based on B97-D/def2-TZVP free energies using the popular $(75,302)$ integration grid can vary from $62: 38$ to $99: 1$ by simply rotating the transition state structures. Relative free energies for the regiocontrolling transition state structures for an $\mathrm{Ir}$-catalyzed $\mathrm{C}-\mathrm{H}$ functionalization reaction computed using M06/6-31G $(d, p) / \mathrm{LANL} 2 \mathrm{DZ}$ and the same grid can vary by more than $5 \mathrm{kcal} \mathrm{mol}^{-1}$, resulting in predicted regioselectivities that range anywhere from 14:86 to >99:1. Errors of these magnitudes occur for different functionals and basis sets, are widespread among modern applications of DFT, and can be reduced by using much denser integration grids than commonly employed.
\end{abstract}




\section{Introduction}

Density functional theory (DFT) is widely used to understand and predict the properties and reactivity of complex organic and organometallic systems. ${ }^{1-9}$ Many of these applications require accuracies in relative free energies $(\Delta \mathrm{G}$ or $\Delta \Delta \mathrm{G})$ that are smaller than the quantities of interest, which are often only a few $\mathrm{kcal} \mathrm{mol}^{-1}$. For instance, in the context of regio- and stereoselective reactions, a $1 \mathrm{kcal} \mathrm{mol}^{-1}$ change in relative free energy barriers can be the difference between modest and high selectivity. ${ }^{3}$ Luckily, modern DFT methods are capable of such accuracy for many systems, and errors arising from poor choices of exchange-correlation functional $^{10}$ and basis set are widely appreciated. However, there is an additional source of potentially significant errors in DFT free energies that goes largely unnoticed.

This other axis of error arises from the choice of integration grid. The evaluation of integrals over the exchange-correlation potential in DFT requires a numerical quadrature scheme, which is accomplished in most quantum chemistry programs using atom-centered grids comprising a direct product of $N^{r}$ radial and $N^{\Omega}$ angular grid points. These integration grids are typically denoted by the number of radial shells and the number of angular points in each shell as $\left(N^{r}, N^{\Omega}\right)$. Integration grid errors can significantly impact energies and other molecular properties. $^{11-26}$ For example, in 2010, Wheeler and Houk ${ }^{22}$ reported grid errors exceeding 5 kcal $\mathrm{mol}^{-1}$ in isomerization energies of small organic molecules when members of the M06 suite of functionals ${ }^{27}$ were paired with the popular SG-1 grid [a pruned $(50,194)$ grid]. ${ }^{28}$ In 2016 , HeadGordon and co-workers ${ }^{24}$ assessed grid errors across a wider array of Minnesota functionals, finding that atomization energies are often converged with a $(75,302)$ grid for all but two of these functionals and fully converged for all them with a $(99,590)$ grid. In light of the grid sensitivity of electronic energies from some DFT functionals, there have been recent efforts to account for this in the development and (re)parameterization of DFT functionals $\mathrm{s}^{23,25-26,29}$ and Herbert et al. ${ }^{30}$ have developed standardized grids designed to provide accurate energies for modern DFT functionals.

These previously documented grid errors primarily concerned electronic energies. Here, we show that popular grids can lead to large errors in free energies, with harrowing implications for DFT studies of organic and organometallic systems. These errors arise from the sensitivity of the entropic component of the free energy to small variations in the values of low-frequency vibrational modes but are distinct from errors due to the treatment of these modes as harmonic 
oscillators. Moreover, they occur using different DFT functionals, basis sets, and quantum chemistry codes and require at least a $(99,590)$ grid to be resolved in most cases.

\section{Results and Discussion}

In the absence of an external field, the energy of a molecule should be independent of its orientation relative to the lab-fixed Cartesian coordinate system. However, because the atomcentered integration grids used in most quantum chemistry packages are anchored to the Cartesian axes, DFT energies typically lack invariance with respect to rigid-body rotations. While this has been known for decades, ${ }^{28,31-33}$ some users are not aware of this shortcoming because many quantum chemistry codes automatically reorient molecules such that the principle axes are aligned with the Cartesian axes (the so-called 'standard orientation'). Luckily, this lack of rotational invariance has negligible impact on relative electronic energies, at least for small systems and some DFT functionals (vide infra). For instance, the B97-D/def2-TZVP electronic energy (E) of 2-butyne relative to 1,3-butadiene (reaction 1) is plotted in Figure 1A as a function of the rotation of 2-butyne around an axis perpendicular to the molecule using the popular $(75,302)$ grid. The variation in this relative energy is $0.01 \mathrm{kcal} \mathrm{mol}^{-1}$.

However, first and second derivatives of the DFT energy with respect to nuclear coordinates also lack rotational invariance. The result is that both the geometries of stationary points and the values of harmonic vibrational frequencies depend on the molecular orientation. For example, in the case of 2-butyne, the B97-D/def2-TZVP value for the methyl rocking mode using with a $(75,302)$ grid varies from $31 i$ and $29 \mathrm{~cm}^{-1}$ upon rotation (see Figure 1B). In other words, the very nature of this stationary point on this DFT potential energy surface depends on the molecular orientation. In terms of zero-point vibrational corrected energies and thermal enthalpies, such vibrational frequency fluctuations are typically inconsequential (if one excludes orientations for which there is an anomalous imaginary mode). On the other hand, the impact on free energies is substantial due to the sensitivity of the entropic component to low-frequency vibrational modes within the standard rigid-rotor/harmonic-oscillator (RRHO) approximation. For example, using a $(75,302)$ grid, and excluding the orientations for which the low-frequency mode is imaginary, there is $\sim 1 \mathrm{kcal} \mathrm{mol}^{-1}$ variation in $\Delta \mathrm{G}$ for reaction 1 (see Figure $1 \mathrm{~A}$ ). Using the SG-1 grid the variation in the free energy of 2-butyne is even larger. 

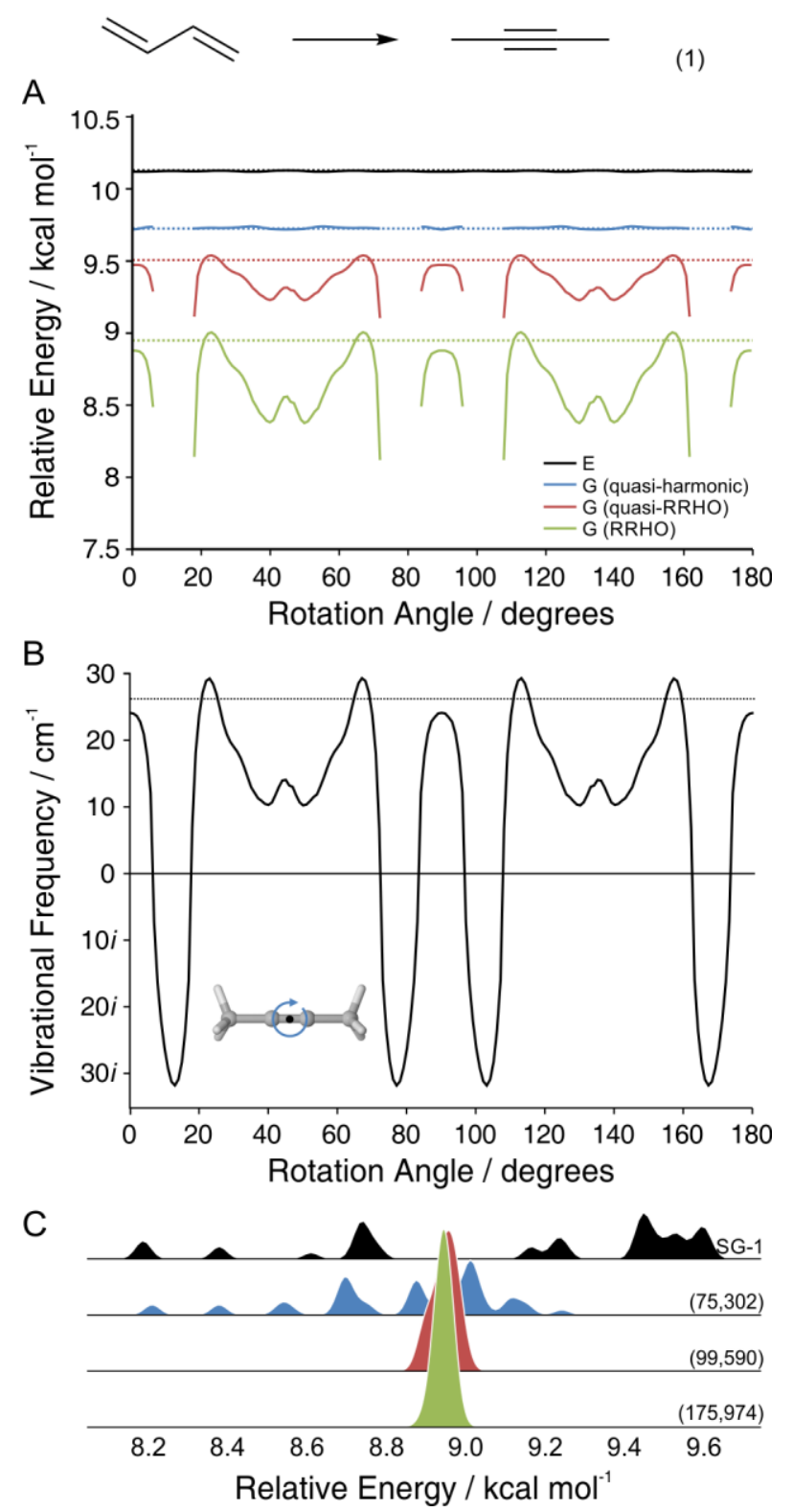

Figure 1. A) B97-D/def2-TZVP energy (E) and free energy $(\mathrm{G})$ of reaction 1 as a function of rotation of $\mathrm{D}_{3 \mathrm{~h}}$-symmetric 2-butyne around the indicated axis using a $(75,302)$ grid. Free energies for orientations at which the low-lying frequency is imaginary are omitted. The grid-converged values are indicated with dotted lines. B) Low-frequency vibration for 2-butyne as a function of the indicated rotation using a $(75,302)$ grid. The grid-converged value is indicated with a dotted line. C) Distribution of RRHO free energies for reaction 1 across 50 representative orientations using four popular integration grids.

Variations in both vibrational frequencies and free energies exhibit a $90^{\circ}$ periodicity (see Figures $1 \mathrm{~A}$ and B), mirroring the octahedral symmetry of the atom-centered grids. ${ }^{34}$ To assess how these errors behave for different grids, the distribution of free energies for reaction 1 across 50 representative orientations of 2-butyne are plotted for four popular integration grids in Figure 
1C. The extent of variations in $\Delta \mathrm{G}$ contracts with increasing grid size, and by the $(99,590)$ grid becomes negligible. In other words, the lack of rotational invariance can be used as a gauge of the grid errors for a given free energy difference. Similar variations occur using other functionals and basis sets [see SI Figure S1 for data using B97-D, ${ }^{35-36}$ B3LYP,${ }^{37}$ PBE,${ }^{38} \omega B$ 97X-D, ${ }^{39}$ and M06-2X ${ }^{40}$ paired with 6-31G(d), cc-pVTZ, and def2-TZVP] $]^{41-42}$ and different electronic structure packages (see SI Figure S2 for data computed using Orca ${ }^{43}$ ).

Of course, the real culprit is the RRHO approximation, which should not be used for lowfrequency vibrational modes. Instead, these modes should be projected out of the hessian and treated separately as, for example, hindered rotors. ${ }^{44-47}$ While such treatments are feasible for simple molecules such as 2-butyne, in which the problematic mode involves simple combinations of internal coordinates, they are impractical for the large systems on which DFT methods are most often applied. ${ }^{48}$ Instead, users tend to employ more approximate treatments of the entropic contribution of low-frequency vibrations. Chief among these are the quasi-harmonic approximation of Cremer and $\operatorname{Truhlar}^{49}$ and the quasi-RRHO approximation of Grimme. ${ }^{50}$ In the former, vibrational frequencies below some cutoff value (typically $100 \mathrm{~cm}^{-1}$ ) are changed to that cutoff value and the RRHO formulae applied. In the latter, the entropic contributions of vibrational frequencies below some cutoff (again, typically $100 \mathrm{~cm}^{-1}$ ) are interpolated between those from an effective free-rotor and the RRHO limit. Figure 1A shows that the use of the quasi-RRHO approach reduces variations in $\Delta \mathrm{G}$ for reaction 1 by limiting the impact of lowfrequency modes. The quasi-harmonic approximation all but eliminates these variations because the most problematic mode is consistently below the cutoff value, so is shifted to a constant value of $100 \mathrm{~cm}^{-1}$. However, grid errors in quasi-harmonic free energies will remain in cases in which one or more vibrational frequencies exhibit variability around the cutoff value (vide infra).

Such grid errors are not isolated to reaction 1 but will occur for any transformation in which one or more molecules exhibit low frequency vibrational modes. ${ }^{51}$ This includes most organic and organometallic molecules studied using DFT. ${ }^{1-9}$ Moreover, variations in DFTcomputed free energies upon rotations can be substantial, resulting in uncertainties in free energies that can overshadow quantities of interest. Scheme 1 shows four systems that highlight the potential impacts of these grid errors, spanning a range of molecular sizes and complexities. Reaction 2 concerns the relative free energy $(\Delta G)$ of the atropisomeric HIV integrase inhibitor 1 and its diastereomer $\left(\mathbf{1}^{\prime}\right),{ }^{52}$ while quantifying the rate of reaction 3 requires the torsional free energy barrier $\left(\Delta \mathrm{G}^{\dot{\ddagger}}\right)$ of $4,4^{\prime}$-dimethyl-1,1'-biphenyl. Predicting the selectivity of reactions 4 and 
5 requires relative free energies $\left(\Delta \Delta \mathrm{G}^{\ddagger}\right)$ for competing transition state (TS) structures leading to alternative products. In particular, the stereoselective addition of allenyltrichlorosilane to benzaldehyde catalyzed by a 2,2'-biquinoline- $N, N^{\prime}$-dioxide ${ }^{53-56}$ (reaction 4) depends on the relative free energy of TS structures leading to enantiomeric products. In reaction 5 , Ir can catalyze the $\mathrm{C}-\mathrm{H}$ functionalization of indole at either $\mathrm{C}_{2}$ or $\mathrm{C}_{7}$ depending on the relative free energy of the corresponding TS structures for the concerted metalation-deprotonation. ${ }^{57}$ Free energy swings of $\pm 2 \mathrm{kcal} \mathrm{mol}^{-1}$ can be catastrophic for predicting the outcome of reactions 4 and 5 given the exponential dependence of selectivities on $\Delta \Delta \mathrm{G}^{\ddagger} .{ }^{3}$ For each of these transformations, we quantified uncertainties in the energy, enthalpy, and free energy due to integration grid errors by sampling representative orientations, fully optimizing the geometry for each orientation (see Computational Methods for details).

Scheme 1. Representative transformations for which accurate relative free energies are required.

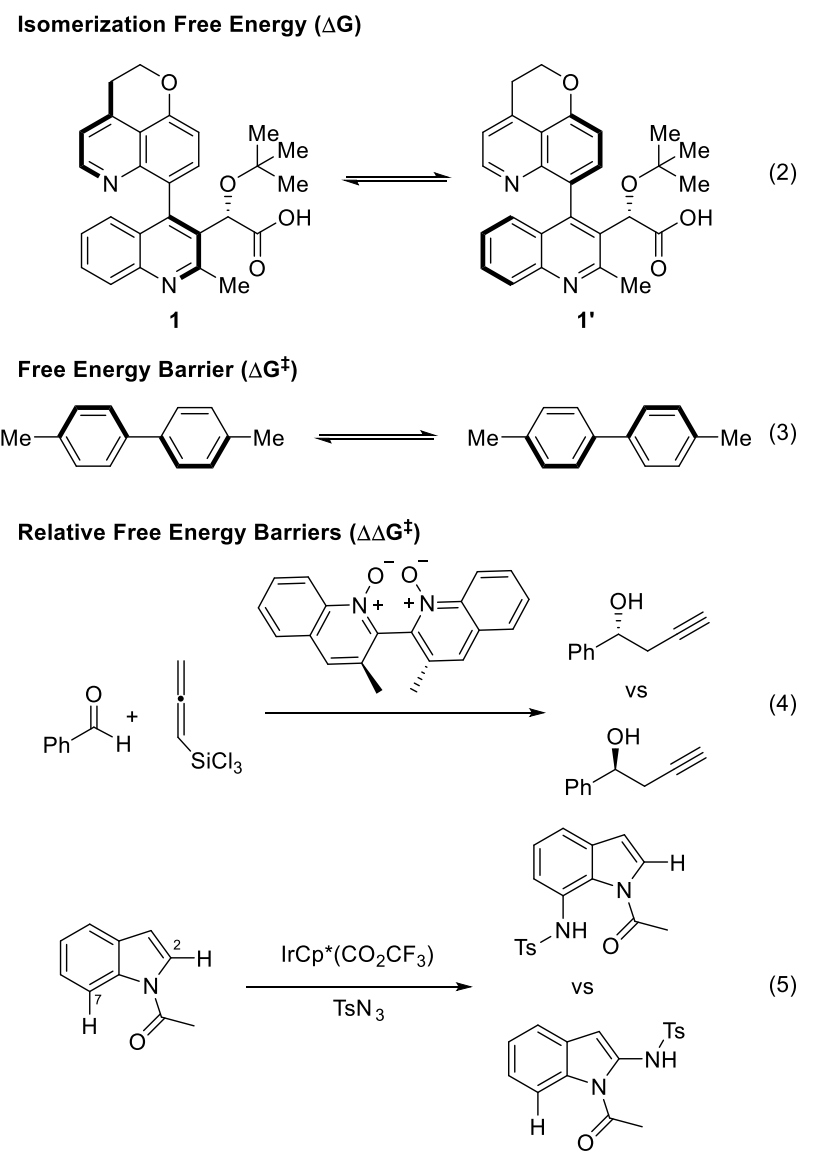


Table 1. Grid converged energy, enthalpy, and free energy differences and minimum and maximum values across representative orientations for four integration grids for reactions $1-5$, in kcal mol ${ }^{-1}$.

\begin{tabular}{|c|c|c|c|c|c|c|c|c|c|}
\hline \multirow[t]{2}{*}{ Reaction } & \multirow{2}{*}{$\begin{array}{c}\text { Grid Converged } \\
\text { Value }^{\mathrm{a}} \\
\end{array}$} & \multicolumn{2}{|c|}{ SG-1 } & \multicolumn{2}{|c|}{$(75,302)$} & \multicolumn{2}{|c|}{$(99,590)$} & \multicolumn{2}{|c|}{$(175,974)$} \\
\hline & & Min & Max & Min & Max & Min & $\operatorname{Max}$ & Min & $\operatorname{Max}$ \\
\hline \multicolumn{10}{|c|}{ Electronic Energy } \\
\hline (1) & 10.1 & 10.1 & 10.2 & 10.1 & 10.1 & 10.1 & 10.1 & 10.1 & 10.1 \\
\hline (2) & 1.1 & 0.7 & 1.5 & 0.9 & 1.2 & 1.1 & 1.1 & 1.1 & 1.1 \\
\hline (3) & 2.0 & 1.6 & 2.2 & 1.9 & 2.0 & 2.0 & 2.0 & 2.0 & 2.0 \\
\hline (4) & 0.5 & -0.2 & 1.2 & 0.3 & 0.8 & 0.5 & 0.6 & 0.5 & 0.5 \\
\hline (5) & 1.3 & 0.1 & 2.0 & 0.9 & 1.6 & 1.2 & 1.4 & 1.3 & 1.3 \\
\hline \multicolumn{10}{|c|}{ Enthalpy $(298 \mathrm{~K})$} \\
\hline (1) & 10.0 & 10.0 & 10.0 & 10.0 & 10.0 & 10.0 & 10.0 & 10.0 & 10.0 \\
\hline (2) & 1.1 & 0.6 & 1.5 & 0.9 & 1.4 & 1.0 & 1.2 & 1.0 & 1.1 \\
\hline (3) & 1.4 & 1.2 & 2.8 & 1.3 & 1.5 & 1.3 & 1.4 & 1.4 & 1.4 \\
\hline (4) & 0.7 & 0.1 & 1.2 & 0.6 & 0.9 & 0.6 & 0.7 & 0.6 & 0.7 \\
\hline (5) & 1.2 & 0.1 & 3.0 & 0.8 & 2.1 & 1.1 & 1.5 & 1.2 & 1.3 \\
\hline \multicolumn{10}{|c|}{ Free Energy (RRHO Approximation) } \\
\hline (1) & 8.9 & 8.2 & 9.6 & 8.4 & 9.2 & 8.9 & 9.0 & 8.9 & 9.0 \\
\hline (2) & 0.7 & -0.6 & 2.8 & 0.0 & 2.4 & 0.5 & 1.5 & 0.4 & 1.1 \\
\hline (3) & 2.6 & 1.0 & 3.3 & 0.8 & 3.5 & 2.4 & 3.1 & 2.5 & 2.8 \\
\hline (4) & 1.4 & -1.1 & 2.6 & 0.3 & 2.6 & 0.8 & 2.1 & 1.0 & 1.8 \\
\hline (5) & 1.3 & -0.7 & 6.2 & -1.1 & 5.2 & 0.4 & 3.5 & 0.6 & 1.7 \\
\hline \multicolumn{10}{|c|}{ Free Energy (Quasi-RRHO Approximation) ${ }^{50}$} \\
\hline (1) & 9.5 & 9.1 & 9.8 & 9.2 & 9.6 & 9.5 & 9.5 & 9.5 & 9.5 \\
\hline (2) & 0.9 & -0.1 & 2.4 & 0.4 & 2.0 & 0.7 & 1.4 & 0.6 & 1.2 \\
\hline (3) & 2.4 & 1.5 & 2.7 & 1.5 & 2.8 & 2.2 & 2.6 & 2.3 & 2.5 \\
\hline (4) & 1.0 & -0.5 & 2.0 & 0.3 & 1.7 & 0.7 & 1.3 & 0.8 & 1.2 \\
\hline (5) & 1.3 & -0.5 & 5.3 & -0.3 & 4.3 & 0.8 & 2.7 & 0.8 & 1.5 \\
\hline \multicolumn{10}{|c|}{ Free Energy (Quasi-harmonic Approximation) $)^{49}$} \\
\hline (1) & 9.7 & 9.6 & 9.8 & 9.7 & 9.7 & 9.7 & 9.7 & 9.7 & 9.7 \\
\hline (2) & 1.0 & 0.1 & 2.3 & 0.7 & 1.8 & 0.9 & 1.5 & 0.8 & 1.3 \\
\hline (3) & 2.4 & 1.6 & 2.8 & 2.3 & 2.6 & 2.4 & 2.5 & 2.4 & 2.5 \\
\hline (4) & 0.8 & -0.3 & 1.8 & 0.3 & 1.3 & 0.6 & 0.9 & 0.6 & 0.9 \\
\hline (5) & 1.2 & -0.6 & 4.7 & -0.1 & 3.9 & 0.6 & 2.3 & 0.8 & 1.4 \\
\hline
\end{tabular}

${ }^{a}$ Rotationally averaged energy differences using a $(175,974)$ grid.

Table 1 contains grid-converged energies, enthalpies, and free energies (using the RRHO, quasi-RRHO, and quasi-harmonic approximations $)^{49-50}$ for reactions 1-5 as well as the minimum and maximum values that can be obtained by varying one or both molecular orientations using four popular integration grids. For several of these reactions there are large variations in the relative electronic energies, particularly using the SG-1 grid. While these variations are due to previously documented grid errors, their implications for predictions of reaction outcomes should not be overlooked. For instance, using the SG-1 grid, the overall sign of the electronic energy difference between the two stereocontrolling TS structures for reaction 4 can vary. In other words, using this grid one cannot confidently predict which of the stereoisomeric products will be preferentially formed. As reported by Head-Gordon and co-workers ${ }^{24}$ for atomization 
energies, these errors in E are essentially converged by the $(99,590)$ grid. Variations in relative enthalpies are slightly larger, and there are troublingly large swings in computed values using both the SG-1 and $(75,302)$ grids. For instance, the relative enthalpy of the regiocontrolling TS structures for reaction 5 can fall anywhere between 0.8 and $2.1 \mathrm{kcal} \mathrm{mol}^{-1}$, precluding any quantitative prediction of regioselectivity.

Variations in free energies are even larger and more slowly convergent with respect to grid size. Moreover, while the quasi-RRHO and quasi-harmonic approximations reduce the ranges of free energy differences, they remain significant (see Table 1). To provide a better feel for the spread of possible computed free energy differences using different popular grids, the distributions of free energy values across different orientations for reactions 2-5 are shown in Figure 2. In all cases, the spread of free energy differences computed using the SG-1 and $(75,302)$ grids are substantial. Moreover, for some of these reactions one can obtain qualitatively different results using a given level of theory by considering different molecular orientations. For example, using a $(75,302)$ grid the computed torsional free energy barrier for reaction 3 can be anywhere from $0.8 \mathrm{kcal} \mathrm{mol}^{-1}$ to $3.5 \mathrm{kcal} \mathrm{mol}^{-1}$, corresponding to two orders of magnitude in terms of reaction rate. Even worse, the relative free energies of the HIV integrase inhibitor 1 relative its diastereomer (reaction 2) can vary from $-1.1 \mathrm{kcal} \mathrm{mol}^{-1}$ to $+2.6 \mathrm{kcal} \mathrm{mol}^{-1}$ using the SG-1 grid. This is particularly important, because it was found experimentally that the biologically active form of this potential drug (1) was the less stable diastereomer, necessitating the development of a complex stereoselective synthesis of the desired molecule. ${ }^{58}$

The variations in $\Delta \Delta \mathrm{G}^{\ddagger}$ for reactions 4 and 5 are particularly large, which does not bode well for predictions of selectivity. For example, for reaction 4 RRHO $\Delta \Delta \mathrm{G}^{\ddagger}$ values using a $(75,302)$ grid range from $0.3 \mathrm{kcal} \mathrm{mol}^{-1}$ to $2.6 \mathrm{kcal} \mathrm{mol}^{-1}$ depending on the TS orientations. In other words, one can predict selectivities for this reaction that range anywhere from 62:38 to 99:1 by choosing different molecular orientations. Using the SG-1 grid, predicted selectivities can range from $14: 86$ to $99: 1$. Even with the $(99,590)$ and $(175,974)$ grids, $\Delta \Delta \mathrm{G}^{\ddagger}$ still varies by 1.2 and $0.8 \mathrm{kcal} \mathrm{mol}^{-1}$, respectively, leading to unacceptably large uncertainties in predicted enantioselectivities for this simple organocatalytic reaction. Variations in the free energy difference between the regiocontrolling TS structures for reaction 5 are even larger, spanning more than $6 \mathrm{kcal} \mathrm{mol}^{-1}$ for the SG-1 and $(75,302)$ grids. For instance, using the $(75,302)$ grid, this free energy difference can fall anywhere from -1.1 to $+5.2 \mathrm{kcal} \mathrm{mol}^{-1}$. Figure $2 \mathrm{~B}$ shows the energy, enthalpy, and RRHO free energy of TS2 in a fixed orientation relative to different 
orientations of TS1 using a $(75,302)$ grid. By varying just one TS structure, $\Delta \Delta \mathrm{G}^{\ddagger}$ values fluctuate wildly between $\pm 1 \mathrm{kcal} \mathrm{mol}^{-1}$, highlighting the sensitivity of DFT free energies to even small changes in orientation. Even with the very large $(174,975)$ grid the $\Delta \Delta \mathrm{G}^{\ddagger}$ values for reaction 5 can vary by more than $1 \mathrm{kcal} \mathrm{mol}^{-1}$.

A

A

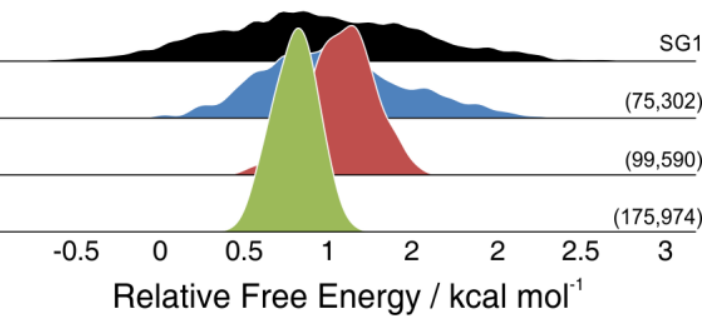

B

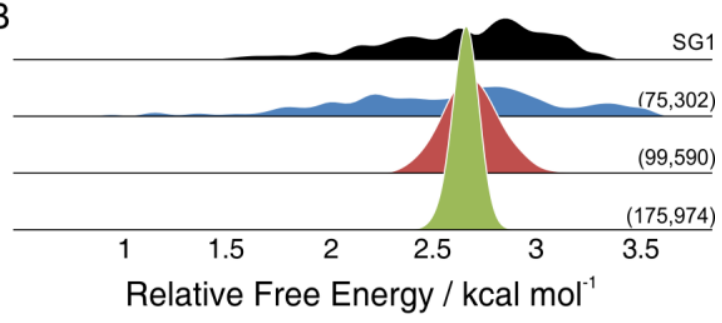

C

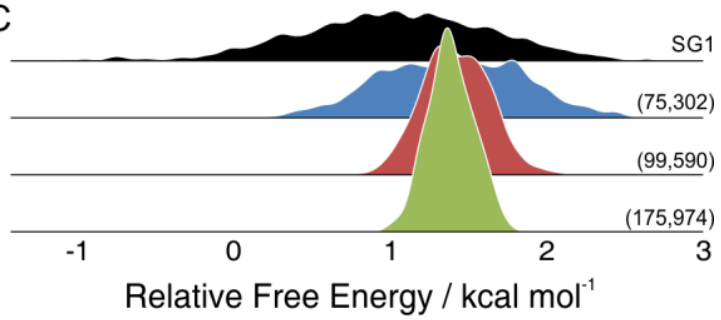

D

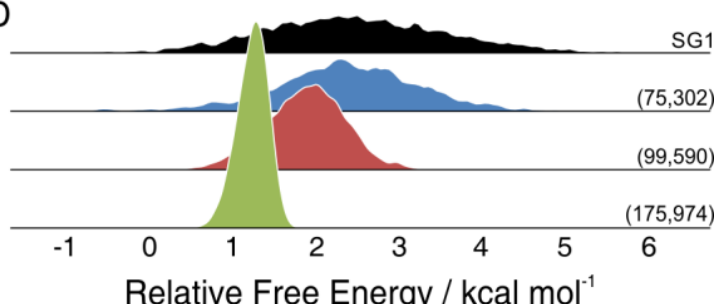

Figure 2. Distribution of RRHO free energy differences across all combinations of sampled orientations for the two structures for reactions (A) 2, (B) 3, (C) 4, and (D) 5 .

The lack of rotational invariance of DFT energies, gradients, and vibrational frequencies is widely acknowledged (although not widely appreciated among users), ${ }^{31-33}$ and documentation for many quantum chemistry packages include caveats regarding the sensitivity of vibrational frequencies to integration grids. For instance, starting with Gaussian98, a $(99,590)$ grid has been recommended for computing 'very low frequency modes.' Similarly, the Orca manual advises 
users to assess the convergence of vibrational frequencies with respect to integration grid. Unfortunately, these sage pieces of advice seem to have gone largely unheeded among users, perhaps because the magnitude of the resulting errors in free energies was not fully appreciated. Hopefully the data in Table 1 will help change this behavior, as a $(99,590)$ grid should be considered a minimal integration grid for the computation of free energies for systems with lowfrequency vibrational modes. Moreover, as shown above, errors in relative free energies approaching $1 \mathrm{kcal} \mathrm{mol}^{-1}$ in can remain even with a much larger $(175,974)$ grid.

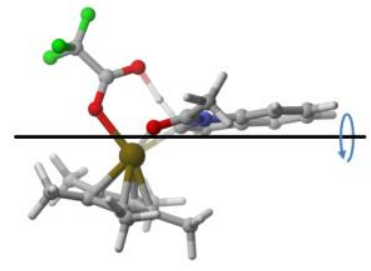

TS1

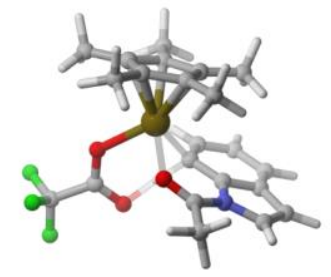

TS2

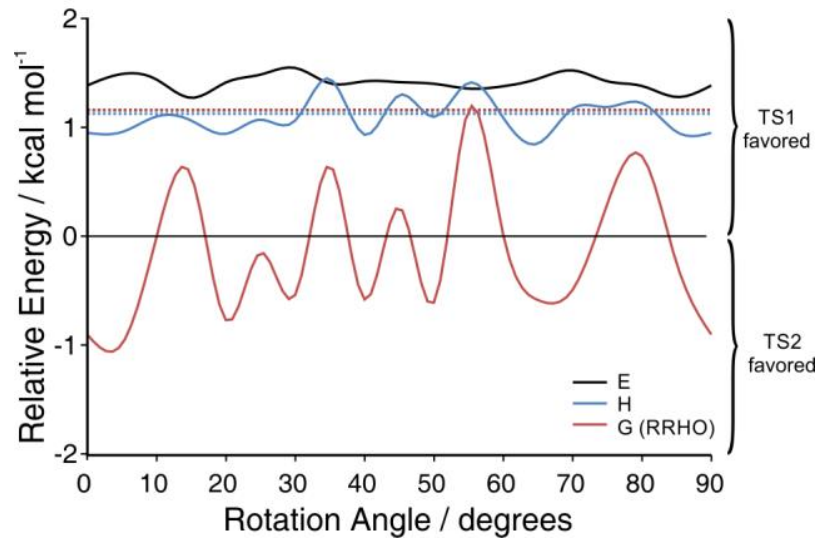

Figure 3. Energy $(\mathrm{E})$, enthalpy $(\mathrm{H})$, and RRHO free energy $(\mathrm{G})$ of TS2 relative to TS1 using a $(75,302)$ grid for different orientations of TS1 and the pictured orientation of TS2 (gridconverged values indicated with dotted lines).

One means of dealing with the lack of rotational invariance is to use the "standard orientation' in all computations. In other words, if the free energy changes when rotating a molecule, then do not rotate it! Equivalently, the atom-centered integration grids can be rotated with the molecule. ${ }^{32}$ Along the same lines, the Orca manual warns against the use of 'grossly different orientations' when computing relative energies of similar molecules. These approaches all rest on the assumption that similar orientations will minimize grid errors in computed free energies. Table 2 lists errors in RRHO free energies for reactions 1-5 using the standard orientation, relative to grid-converged values, for four popular grids. In most cases, the errors in the standard orientation are large, particularly for the smaller grids. For example, using the SG-1 
grid, $\Delta \Delta \mathrm{G}^{\ddagger}$ for reaction 5 is $1.5 \mathrm{kcal} \mathrm{mol}^{-1}$ from the grid-converged value of $1.3 \mathrm{kcal} \mathrm{mol}^{-1}$. While the errors are smaller for the other reactions and grids, they are not negligible until reaching the $(175,974)$ grid. In other words, the use of a standard orientation (or equivalently, rotating the atom-centered grids with the molecule) does not minimize these grid errors. Similarly, the use of 'similar orientations' does not necessarily lead to error cancelation. This can be seen explicitly in Figure 4, which shows the relative free energies of the two regiocontrolling TS structures for reaction 5 as they are rotated simultaneously around the indicated axes starting from their respective standard orientations. Even using a $(99,590)$ grid, there is more than $2 \mathrm{kcal}$ $\mathrm{mol}^{-1}$ variation in the free energy difference between these regiocontrolling TS structures.

Table 2. Errors in RRHO free energy differences for reactions 1-5 using the standard orientation, relative to grid-converged values, in $\mathrm{kcal} \mathrm{mol}^{-1}$.

\begin{tabular}{ccccc}
\hline Reaction & SG-1 & $(75,302)$ & $(99,590)$ & $(175,974)$ \\
\hline$(1)$ & 0.5 & -0.1 & 0.0 & 0.0 \\
$(2)$ & 0.8 & -0.1 & 0.2 & -0.1 \\
$(3)$ & 0.0 & -0.8 & -0.1 & 0.0 \\
$(4)$ & -0.9 & -0.4 & 0.3 & 0.0 \\
$(5)$ & 1.5 & 0.6 & 0.6 & -0.2 \\
\hline
\end{tabular}
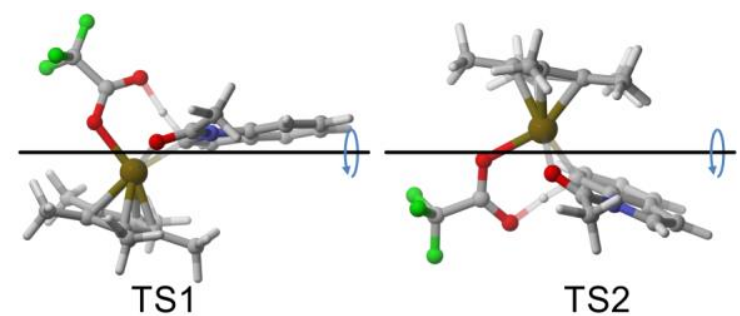

TS2

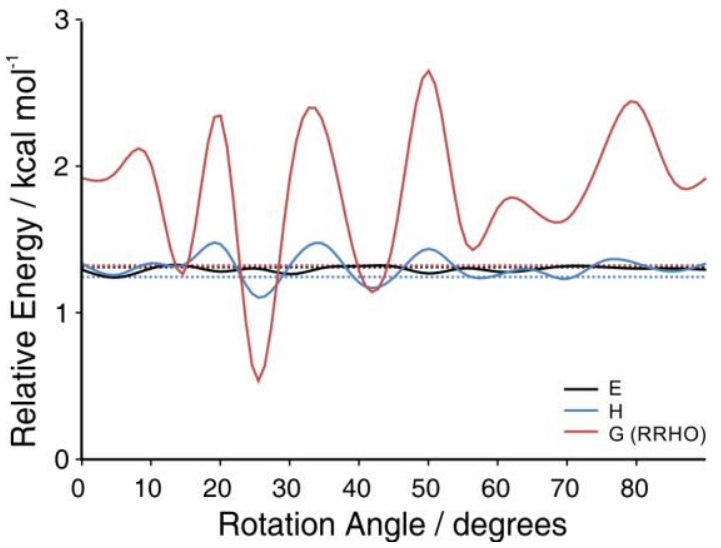

Figure 4. Energy (E), enthalpy (H), and RRHO free energy (G) of TS2 relative to TS1 for simultaneous rotation of TS1 and TS2 around the indicated axis starting from the standard orientation (pictured) using a $(99,590)$ grid. Grid-converged values indicated with dotted lines. 


\section{Conclusions}

The evaluation of accurate relative free energies is vital for many applications of quantum chemistry to organic and organometallic systems. ${ }^{1-9}$ We showed that the lack of rotational invariance of commonly employed integration grids results in large uncertainties in DFTcomputed free energy differences. Based on data from a small set of examples (see Scheme 1 and Table 1), the popular SG-1 and $(75,302)$ grids can lead to errors exceeding $5 \mathrm{kcal} \mathrm{mol}^{-1}$ in relative free energies for many systems. While the reactions in Scheme 1 were chosen to highlight these errors, they are not atypical. Consequently, errors of this magnitude are likely widespread among modern DFT applications and can easily overshadow chemically meaningful free energy differences. The data show that the outcomes of many organic and organometallic reactions predicted using a given level of DFT theory can vary qualitatively depending on molecular orientation. This is a problem, and results in the troubling situation whereby DFT results can be 'tuned' over a wide range by varying molecular orientations. ${ }^{59}$ This raises sobering questions about the vast body of computational studies of stereo- and regioselective reactions based on DFT-computed free energies, including a great deal of work from our group. For instance, how many cases in which computed free energy differences agreed with experimental selectivity data were a result of grid errors fortuitously cancelling other errors? Alternatively, perhaps the reactions for which DFT-predicted selectivities did not match experiment were due to a poor choice of molecular orientations. ${ }^{60}$ These data also explain why relative enthalpies often provide better agreement with experimental selectivities than ostensibly more physically meaningful free energies. ${ }^{61}$

The underlying source of these errors is the unphysically large contribution of lowfrequency vibrational modes to the entropic component of the free energy in the standard RRHO treatment. To this end, the quasi-harmonic and quasi-RRHO approximations ${ }^{49-50}$ reduce, but do not eliminate, these grid errors. At the same time, despite continued advances in more rigorous treatments of the entropic contributions of low-frequency vibrational modes, ${ }^{62-63}$ such approaches are still impractical for the organic and organometallic systems most often subject to DFT computations. Baik et $a .^{64}$ recently warned of the unphysical contributions of low frequency vibrational modes to the free energies in organometallic systems, showing that rotations of a $\mathrm{Cp}$ ring in a $\mathrm{Rh}$-complex can lead to variations of nearly $2 \mathrm{kcal} \mathrm{mol}^{-1}$ in the free energy. The grid errors discussed above are arguably more alarming, because they concern larger variations in free energies for rotations of the entire molecule. 
The good news is that these errors are reduced substantially using a $(99,590)$ grid in many cases. Consequently, this grid (which is the default in Gaussian16) ${ }^{65}$ should be considered a minimal grid when using DFT to compute free energies any system with low-frequency vibrational modes $\left(<100 \mathrm{~cm}^{-1}\right)$. However, given that errors of $\sim 1 \mathrm{kcal} \mathrm{mol}^{-1}$ remain for some systems even with a $(175,974)$ grid, we implore users to compute free energies for several molecular orientations using multiple integration grids in order to gauge the magnitude of grid errors and to guide the choice of the most appropriate integration grid for a given problem.

\section{Theoretical Methods}

Gas-phase energies, enthalpies (298K), and free energies (298K) for each energy minimum or TS structure for reactions 1-5 were computed for 25 (for reactions 2-4) or 50 (for reactions 1 and 5) representative orientations using four integration grids: (50,194), (75,302), $(99,590)$, and $(175,974)$ [the latter uses a $(250,974)$ grid for Ir]. These orientations were generated by performing independent rigid body rotations around the three principle axes in $10^{\circ}$ (or $5^{\circ}$ ) increments up to $80^{\circ}$ (or $85^{\circ}$ ) starting from the respective standard orientations using AaronTools. ${ }^{66}$ For reaction 1, only the orientation of 2-butyne was varied, because the free energy of 1,3-butadiene is essentially constant across different orientations. For each orientation, the geometry was fully optimized using the corresponding integration grid such that the maximum and RMS gradients were below $1.5 \times 10^{-5}$ and $1.0 \times 10^{-5}$ hartree/bohr, respectively. Harmonic vibrational frequencies were then computed via analytic second derivatives. It should be noted that if geometries were not reoptimized at each orientation, the observed variations in enthalpies and free energies were even larger and there were often many superfluous imaginary vibrational modes. Grid-converged values of energies, enthalpies, and free energies were obtained by averaging the corresponding quantity using a $(175,974)$ grid across all combinations of sampled orientations. In Table 1, the minimum and maximum energy differences correspond the extreme energy differences across all sampled orientations. For orientations for which the correct rotational symmetry number was not automatically recognized, we manually appended the corresponding correction to the entropy. Quasi-RRHO and quasi-harmonic free energies were computed using AaronTools. ${ }^{66}$

Data for reactions 1-4 were computed at the B97-D/def2-TZVP level of theory using density fitting. It should be noted that the documented grid errors are independent of whether density fitting is used (see SI Figure S3). Data for reaction 5 were computed using M06 paired 
with the LANL2DZ/HayWadt basis set/ECP on $\mathrm{Ir}$ and the $6-31 \mathrm{G}(d, p)$ basis set on all other atoms, following Ref. 57. For reaction 4, initial TS structures were taken from Ref. 54; initial TS structures for reaction 5 were taken from Ref. 57. Predicted selectivities for reactions 4 and 5 assume Curtin-Hammett conditions. The computations presented above were performed using

Gaussian09; ${ }^{67}$ selected data for 2-butyne were also computed using Orca. ${ }^{43}$ Molecular structure figures were generated using CYLView. ${ }^{68}$

\section{Acknowledgments}

This work was supported by the National Science Foundation (Grant CHE-1665407) and conducted with high performance computing resources provided by the Georgia Advanced Computing Resource Center (http://gacrc.uga.edu). We thank D. Maziotti, R. S. Paton, P.-O. Norrby, C. E. Webster, M. L. Coote, and J. M. Herbert for fruitful discussions. A. J. Schaefer is thanked for assistance regarding reaction 5 .

Supporting Information Available: Additional computational data and figures, absolute energies, Cartesian coordinates.

\section{References}

1. Houk, K. N.; Cheong, P. H., Computational prediction of small-molecule catalysts. Nature 2008, 455, 309-313.

2. Sperger, T.; Sanhueza, I. A.; Kalvet, I.; Schoenebeck, F., Computational Studies of Synthetically Relevant Homogeneous Organometallic Catalysis Involving Ni, Pd, Ir, and Rh: An Overview of Commonly Employed DFT Methods and Mechanistic Insights. Chem. Rev. 2015, 115, 9532-9586.

3. Peng, Q.; Duarte, F.; Paton, R. S., Computing organic stereoselectivity - from concepts to quantitative calculations and predictions. Chem. Soc. Rev. 2016, 45, 6093-6107.

4. Lam, Y. H.; Grayson, M. N.; Holland, M. C.; Simon, A.; Houk, K. N., Theory and Modeling of Asymmetric Catalytic Reactions. Acc. Chem. Res. 2016, 49, 750-762.

5. Sperger, T.; Sanhueza, I. A.; Schoenebeck, F., Computation and Experiment: A Powerful Combination to Understand and Predict Reactivities. Acc. Chem. Res. 2016, 49, 1311-1319.

6. Houk, K. N.; Liu, F., Holy Grails for Computational Organic Chemistry and Biochemistry. Acc. Chem. Res. 2017, 50, 539-543.

7. Poree, C.; Schoenebeck, F., A Holy Grail in Chemistry: Computational Catalyst Design: Feasible or Fiction? Acc. Chem. Res. 2017, 50, 605-608.

8. Ahn, S.; Hong, M.; Sundararajan, M.; Ess, D. H.; Baik, M. H., Design and Optimization of Catalysts Based on Mechanistic Insights Derived from Quantum Chemical Reaction Modeling. Chem. Rev. 2019, 119, 6509-6560. 
9. Maji, R.; Mallojjala, S. C.; Wheeler, S. E., Chiral phosphoric acid catalysis: from numbers to insights. Chem. Soc. Rev. 2018, 47, 1142-1158.

10. Goerigk, L.; Mehta, N., A Trip to the Density Functional Theory Zoo: Warnings and Recommendations for the User*. Aust. J. Chem. 2019.

11. Termath, V.; Sauer, J., Optimized molecular integration schemes for density functional theory ab initio molecular dynamics simulations. Chem. Phys. Lett. 1996, 255, 187-194.

12. Dressler, S.; Thiel, W., Anharmonic force fields from density functional theory. Chem. Phys. Lett. 1997, 273, 71-78.

13. Martin, J. M. L.; Bauschlicher, C. W.; Ricca, A., On the integration accuracy in molecular density functional theory calculations using Gaussian basis sets. Comput. Phys. Commun. 2001, 133, 189-201.

14. Johnson, E. R.; Wolkow, R. A.; DiLabio, G. A., Chem. Phys. Lett. 2004, 394, 334-338.

15. Papas, B. N.; Schaefer, H. F., Concerning the precision of standard density functional programs: GAUSSIAN, MOLPRO, NWCHEM, Q-CHEM, and GAMESS. Journal of Molecular Structure-Theochem 2006, 768, 175-181.

16. Grafenstein, J.; Izotov, D.; Cremer, D., Avoiding singularity problems associated with metaGGA (generalized gradient approximation) exchange and correlation functionals containing the kinetic energy density. J. Chem. Phys. 2007, 127, 214103.

17. Grafenstein, J.; Cremer, D., Efficient density-functional theory integrations by locally augmented radial grids. J. Chem. Phys. 2007, 127, 164113.

18. Jiménez-Hoyos, C. A.; Janesko, B. G.; Scuseria, G. E., Evaluation of range-separated hybrid density functionals for the prediction of vibrational frequencies, infrared intensities, and Raman activitiesw. Phys. Chem. Chem. Phys. 2008, 10, 6621-6629.

19. Johnson, E. R.; Becke, A. D.; Sherrill, C. D.; DiLabio, G. A., Oscillations in metageneralized-gradient approximation potential energy surfaces for dispersion-bound complexes. J. Chem. Phys. 2009, 131, 034111.

20. Fusti-Molnar, L.; He, X.; Wang, B.; Merz, K. M., Jr., Further analysis and comparative study of intermolecular interactions using dimers from the S22 database. J. Chem. Phys. 2009, 131, 065102.

21. Csonka, G. I.; French, A. D.; Johnson, G. P.; Stortz, C. A., Evaluation of Density Functionals and Basis Sets for Carbohydrates. J. Chem. Theory Comput. 2009, 5, 679-692.

22. Wheeler, S. E.; Houk, K. N., Integration Grid Errors for Meta-GGA-Predicted Reaction Energies: Origin of Grid Errors for the M06 Suite of Functionals. J. Chem. Theory Comput. 2010, 6, 395-404.

23. Mardirossian, N.; Head-Gordon, M., omegaB97X-V: a 10-parameter, range-separated hybrid, generalized gradient approximation density functional with nonlocal correlation, designed by a survival-of-the-fittest strategy. Phys. Chem. Chem. Phys. 2014, 16, 9904-9924.

24. Mardirossian, N.; Head-Gordon, M., How Accurate Are the Minnesota Density Functionals for Noncovalent Interactions, Isomerization Energies, Thermochemistry, and Barrier Heights Involving Molecules Composed of Main-Group Elements? J. Chem. Theory Comput. 2016, 12, 4303-4325.

25. Mardirossian, N.; Head-Gordon, M., omegaB97M-V: A combinatorially optimized, rangeseparated hybrid, meta-GGA density functional with VV10 nonlocal correlation. J. Chem. Phys. 2016, 144, 214110. 
26. Wang, Y.; Jin, X.; Yu, H. S.; Truhlar, D. G.; He, X., Revised M06-L functional for improved accuracy on chemical reaction barrier heights, noncovalent interactions, and solid-state physics. Proc. Natl. Acad. Sci. U. S. A. 2017, 114, 8487-8492.

27. Zhao, Y.; Truhlar, D. G., The M06 Suite of Density Functionals for Main Group Thermochemistry, Thermochemical Kinetics, Noncovalent interactions, Excited States, and Transition Elements: Two New Functionals and Systematic Testing of Four M06 Functionals and Twelve Other Functionals. Theo. Chem. Acc. 2008, 120, 215-241.

28. Gill, P. M. W.; Johnson, B. G.; Pople, J. A., A Standard Grid for Density-Functional Calculations. Chem. Phys. Lett. 1993, 209, 506-512.

29. Wang, Y.; Verma, P.; Jin, X.; Truhlar, D. G.; He, X., Revised M06 density functional for main-group and transition-metal chemistry. Proc. Natl. Acad. Sci. U. S. A. 2018, 115, 1025710262.

30. Dasgupta, S.; Herbert, J. M., Standard grids for high-precision integration of modern density functionals: SG-2 and SG-3. J. Comput. Chem. 2017, 38, 869-882.

31. Jones, R. S.; Mintmire, J. W.; Dunlap, B. I., Geometry Optimization Using Local-Density Functional Methods - Numerical Aspects. Int. J. Quantum Chem. 1988, 34, 77-84.

32. Johnson, B. G.; Gill, P. M. W.; Pople, J. A., A Rotationally Invariant Procedure for DensityFunctional Calculations. Chem. Phys. Lett. 1994, 220, 377-384.

33. Johnson, B. G., Analytic second derivatives of the gradient-corrected density functional energy. Effect of quadrature weight derivatives. Chem. Phys. Lett. 1993, 216, 133-140.

34. Lebedev, V. I.; Laikov, D. N., Dokl. Math. 1999, 59, 477.

35. Grimme, S., Semiempirical GGA-type density functional constructed with a long-range dispersion correction. J. Comput. Chem. 2006, 27, 1787-1799.

36. Becke, A., Density-Functional Thermochemistry. V. Systematic Optimization of ExchangeCorrelation Functionals. J. Chem. Phys. 1997, 107, 8554-8560.

37. Becke, A. D., Density-functional thermochemistry. III. The role of exact exchange. J. Chem. Phys. 1993, 98, 5648-5652.

38. Perdew, J. P.; Burke, K.; Ernzerhof, M., Generalized Gradient Approximation Made Simple. Phys. Rev. Lett. 1996, 77, 3865-3868.

39. Chai, J. D.; Head-Gordon, M., Long-range corrected hybrid density functionals with damped atom-atom dispersion corrections. Phys. Chem. Chem. Phys. 2008, 10, 6615-6620.

40. Zhao, Y.; Truhlar, D. G., The M06 suite of density functionals for main group thermochemistry, thermochemical kinetics, noncovalent interactions, excited states, and transition elements: two new functionals and systematic testing of four M06-class functionals and 12 other functionals. Theor. Chem. Acc. 2008, 120, 215-241.

41. Dunning, T. H., Gaussian basis sets for use in correlated molecular calculations. I. The atoms boron through neon and hydrogen. J. Chem. Phys. 1989, 90, 1007-1023.

42. Weigend, F.; Ahlrichs, R., Balanced basis sets of split valence, triple zeta valence and quadruple zeta valence quality for $\mathrm{H}$ to Rn: Design and assessment of accuracy. Phys. Chem. Chem. Phys. 2005, 7, 3297-3305.

43. Neese, F., Software update: the ORCA program system, version 4.0. Wiley Interdisciplinary Reviews-Computational Molecular Science 2018, 8.

44. McClurg, R. B., Comment on: "The hindered rotor density-of-states interpolation function" [J. Chem. Phys. 106, 6675 (1997)] and "The hindered rotor density- of-states" [J. Chem. Phys. 108, 2314 (1998)]. J. Chem. Phys. 1999, 111, 7163-7164. 
45. McClurg, R. B.; Flagan, R. C.; Goddard Iii, W. A., The hindered rotor density-of-states interpolation function. J. Chem. Phys. 1997, 106, 6675-6680.

46. Ayala, P. Y.; Schlegel, H. B., Identification and treatment of internal rotation in normal mode vibrational analysis. J. Chem. Phys. 1998, 108, 2314-2325.

47. Chuang, Y.-Y.; Truhlar, D. G., Statistical thermodynamics of bond torsional modes. J. Chem. Phys. 2000, 112, 1221-1228.

48. Chief among the reasons this is impractical for larger systems is that the low-frequency vibrational modes for many of these systems are large-amplitude 'rocking motions' that cannot be captured by simple combinations of internal coordiantes. The result is that both the evaluation of the corresponding potential and the corresponding Wilson B-matrix elements is at best cumbersome.

49. Ribeiro, R. F.; Marenich, A. V.; Cramer, C. J.; Truhlar, D. G., Use of solution-phase vibrational frequencies in continuum models for the free energy of solvation. J. Phys. Chem. B 2011, 115, 14556-14562.

50. Grimme, S., Supramolecular binding thermodynamics by dispersion-corrected density functional theory. Chemistry 2012, 18, 9955-9964.

51. Throughout, we consider any vibrational frequency below $100 \mathrm{~cm}-1$ to be a 'low frequency vibrational mode.'

52. Haddad, N.; Mangunuru, H. P. R.; Fandrick, K. R.; Qu, B.; Sieber, J. D.; Rodriguez, S.; Desrosiers, J. N.; Patel, N. D.; Lee, H.; Kurouski, D.; Grinberg, N.; Yee, N. K.; Song, J. H. J.; Senanayake, C. H., Reengineered BI-DIME Ligand Core Based on Computer Modeling to Increase Selectivity in Asymmetric Suzuki-Miyaura Coupling for the Challenging Axially Chiral HIV Integrase Inhibitor. Adv. Synth. Catal. 2016, 358, 3522-3527.

53. Lu, T.; Zhu, R.; An, Y.; Wheeler, S. E., Origin of enantioselectivity in the propargylation of aromatic aldehydes catalyzed by helical N-oxides. J. Am. Chem. Soc. 2012, 134, 3095-3102.

54. Doney, A. C.; Rooks, B. J.; Lu, T. X.; Wheeler, S. E., Design of Organocatalysts for Asymmetric Propargylations through Computational Screening. ACS Catal. 2016, 6, 79487955.

55. Wheeler, S. E.; Seguin, T. J.; Guan, Y.; Doney, A. C., Noncovalent Interactions in Organocatalysis and the Prospect of Computational Catalyst Design. Acc. Chem. Res. 2016, 49, 1061-1069.

56. Nakajima, M.; Saito, M.; Hashimoto, S., Selective synthesis of optically active allenic and homopropargylic alcohols from propargyl chloride. Tetrahedron-Asymmetry 2002, 13, 24492452.

57. Kim, Y.; Park, Y.; Chang, S., Delineating Physical Organic Parameters in Site-Selective C-H Functionalization of Indoles. ACS Cent Sci 2018, 4, 768-775.

58. Fandrick, K. R.; Li, W.; Zhang, Y.; Tang, W.; Gao, J.; Rodriguez, S.; Patel, N. D.; Reeves, D. C.; Wu, J. P.; Sanyal, S.; Gonnella, N.; Qu, B.; Haddad, N.; Lorenz, J. C.; Sidhu, K.; Wang, J.; Ma, S.; Grinberg, N.; Lee, H.; Tsantrizos, Y.; Poupart, M. A.; Busacca, C. A.; Yee, N. K.; Lu, B. Z.; Senanayake, C. H., Concise and Practical Asymmetric Synthesis of a Challenging Atropisomeric HIV Integrase Inhibitor. Angew Chem Int Ed Engl 2015, 54, 7144-7148.

59. This also raises concerns about reproduceability, since one must not only specify the exchange-correlation functional, basis set, and grid, but also the orientation in order for DFTcomputed free energies to be reproduceable.

60. This comment is meant sarcastically. 
61. Rooks, B. J.; Haas, M. R.; Sepulveda, D.; Lu, T. X.; Wheeler, S. E., Prospects for the Computational Design of Bipyridine N,N '-Dioxide Catalysts for Asymmetric Propargylation Reactions. ACS Catal. 2015, 5, 272-280.

62. Jasper, A. W.; Gruey, Z. B.; Harding, L. B.; Georgievskii, Y.; Klippenstein, S. J.; Wagner, A. F., Anharmonic Rovibrational Partition Functions for Fluxional Species at High

Temperatures via Monte Carlo Phase Space Integrals. J. Phys. Chem. A 2018, 122, 1727 1740.

63. Wu, J.; Ning, H.; Xu, X.; Ren, W., Accurate entropy calculation for large flexible hydrocarbons using a multi-structural 2-dimensional torsion method. Phys. Chem. Chem. Phys. 2019, 21, 10003-10010.

64. Ryu, H.; Park, J.; Kim, H. K.; Park, J. Y.; Kim, S. T.; Baik, M. H., Pitfalls in Computational Modeling of Chemical Reactions and How To Avoid Them. Organometallics 2018, 37, 3228-3239.

65. Frisch, M. J.; Trucks, G. W.; Schlegel, H. B.; Scuseria, G. E.; Robb, M. A.; Cheeseman, J. R.; Scalmani, G.; Barone, V.; Petersson, G. A.; Nakatsuji, H.; Li, X.; Caricato, M.; Marenich, A. V.; Bloino, J.; Janesko, B. G.; Gomperts, R.; Mennucci, B.; Hratchian, H. P.; Ortiz, J. V.; Izmaylov, A. F.; Sonnenberg, J. L.; Williams; Ding, F.; Lipparini, F.; Egidi, F.; Goings, J.; Peng, B.; Petrone, A.; Henderson, T.; Ranasinghe, D.; Zakrzewski, V. G.; Gao, J.; Rega, N.; Zheng, G.; Liang, W.; Hada, M.; Ehara, M.; Toyota, K.; Fukuda, R.; Hasegawa, J.; Ishida, M.; Nakajima, T.; Honda, Y.; Kitao, O.; Nakai, H.; Vreven, T.; Throssell, K.; Montgomery Jr., J. A.; Peralta, J. E.; Ogliaro, F.; Bearpark, M. J.; Heyd, J. J.; Brothers, E. N.; Kudin, K. N.; Staroverov, V. N.; Keith, T. A.; Kobayashi, R.; Normand, J.; Raghavachari, K.; Rendell, A. P.; Burant, J. C.; Iyengar, S. S.; Tomasi, J.; Cossi, M.; Millam, J. M.; Klene, M.; Adamo, C.; Cammi, R.; Ochterski, J. W.; Martin, R. L.; Morokuma, K.; Farkas, O.; Foresman, J. B.; Fox, D. J. Gaussian 16 Rev. B.01, Wallingford, CT, 2016.

66. Guan, Y.; Ingman, V. M.; Rooks, B. J.; Wheeler, S. E., AARON: An Automated Reaction Optimizer for New Catalysts. J. Chem. Theory Comput. 2018, 14, 5249-5261.

67. Frisch, M.; Trucks, G.; Schlegel, H.; Scuseria, G.; Robb, M.; Cheeseman, J.; Scalmani, G.; Barone, V.; Mennucci, B.; Petersson, G.; Nakatsuji, H.; Caricato, M.; Li, X.; Hratchian, H.; Izmaylov, A.; Bloino, J.; Zheng, G.; Sonnenberg, J.; Hada, M.; Ehara, M.; Toyota, K.; Fukuda, R.; Hasegawa, J.; Ishida, M.; Nakajima, T.; Honda, Y.; Kitao, O.; Nakai, H.; Vreven, T.; Montgomery, J., JA; Peralta, J.; Ogliaro, F.; Bearpark, M.; Heyd, J.; Brothers, E.; Kudin, K.; Staroverov, V.; Keith, T.; Kobayashi, R.; Normand, J.; Raghavachari, K.; Rendell, A.; Burant, J.; Iyengar, S.; Tomasi, J.; Cossi, M.; Rega, N.; Millam, J.; Klene, M.; Knox, J.; Cross, J.; Bakken, V.; Adamo, C.; ramillo, J.; Gomperts, R.; Stratmann, R.; Yazyev, O.; Austin, A.; Cammi, R.; Pomelli, C.; Ochterski, J.; Martin, R.; Morokuma, K.; Zakrzewski, V.; Voth, G.; Salvador, P.; Dannenberg, J.; Dapprich, S.; Daniels, A.; Farkas, O.; Foresman, J.; Ortiz, J.; Cioslowski, J.; Fox, D. Gaussian 09, Revision D.01, Gaussian, Inc.: 2009.

68. Legault, C. Y. CYLview, 1.0b, Université de Sherbrooke: 2009. 